\title{
The supplementation with Resveratrol and $\alpha$-Tocopherol could reduce the risk of sarcopenia in dogs by counteracting the oxidative stress
}

\author{
Raúl Francisco Pastor ${ }^{\mathrm{a}, *}$, Patrizia Restani ${ }^{\mathrm{b}}$, Juan E. Romero ${ }^{\mathrm{c}}$, Enzo B. Ferro ${ }^{\mathrm{d}}$, \\ Diego R. Serantes ${ }^{\mathrm{e}}$, Juan Ugalde ${ }^{\mathrm{e}}$, Elena Pastor ${ }^{\mathrm{a}}$ and Roberto H. Iermoli ${ }^{\mathrm{f}}$ \\ a Polyphenols, Wine and Health Unit, Fourth Chair of Medicine Universidad de Buenos Aires, \\ Buenos Aires, Argentina \\ ${ }^{\mathrm{b}}$ Departments of Pharmacological and Biomolecular Sciences Scienze, Università degli Studi \\ di Milano, Milano, Italia \\ ${ }^{\mathrm{c}}$ Faculty of Veterinary Sciences of the University of The Pampa, General Pico, La Pampa, Argentina \\ ${ }^{\mathrm{d}}$ High School of Veterinary Sciences, National University of the Center of Buenos Aires Province, \\ University Campus, Buenos Aires, Argentina \\ ${ }^{\mathrm{e}}$ Institute of Biotechnology Research, National University of San Martín, Buenos Aires, Argentina \\ ${ }^{\mathrm{f}}$ Fourth Chair of Medicine, Clinics Hospital University of Buenos Aires, Buenos Aires, Argentina
}

\begin{abstract}
Several scientific evidences show that oxidative stress is associated with the origin, progression and severity of chronic noncommunicable diseases. Due to the longer duration of life, sarcopenia, osteopenia, osteoporosis and cachexia are considered emerging syndromes for dogs and cats. The objective was to investigate the impact of a supplementation with resveratrol and $\alpha$-tocopherol (JUVENIA ${ }^{\circledR}$ ) on the harmful effects of oxidative stress in adult dogs. The study was able to demonstrate this supplementation was able to positively modulate creatine phosphokinase and alkaline phosphatase, which are considered useful biomarkers for sarcopenia, bone formation and speed in bone remodeling. As a result of the longer life of domestic animals and, in particular dogs, the use of food supplements with antioxidant activity could play a critical role in reducing/delaying the onset of chronic-degenerative diseases. On the basis of the present study, the use of products containing resveratrol and $\alpha$-tocopherol acetate seems to be a promising approach for improving the health of dogs.
\end{abstract}

keywords: Oxidative stress, resveratrol, sarcopenia, bone remodeling, $8(\mathrm{OH}) \mathrm{dG}$, dietary supplement.

\section{Introduction}

The incidence of cardiovascular, metabolic, neurodegenerative diseases and cancer has increased significantly in the last ten years [1] and it has been shown that the oxidative stress is one of the most important risk factors in the development of these pathologies $[2,3]$. The metabolic processes depend on the permanent contribution of energy through the

\footnotetext{
*Corresponding author: Raúl Francisco Pastor Tel.: +54 911 4411 4806. E-mail: rpastor@fmed.uba.ar.
}

synthesis of ATP, by mitochondrial oxidative phosphorylation. The obtained ATP is the energy carrier for all cellular systems, but mitochondria in the same biological activity produce more than $95 \%$ of reactive oxygen species and free radicals present in the organism [4].

The chemical instability of free radicals, which have one or two unpaired electron in its external atomic orbital, is responsible for their strong oxidative properties versus all biological molecules, such as DNA, RNA, proteins, carbohydrates and structural lipids, representing the starting point for most chronic 
diseases [5]. During the evolution of the species, biological systems developed a battery of enzymatic and non-enzymatic antioxidant tools in order to counteract the dangerous effects of free radicals. This evolutionary adaptation is not always enough to protect the biological molecules, since the redox balance can shift towards oxidative stress [6].

It is kown that the bone mass and ability to repair fractures in humans decreases after 30 years of age [7]. These parameters are inversely associated with the oxidative stress in both animals and humans $[8,9]$.

The incidence of non-infectious diseases is increasing also in pets; among them, sarcopenia, osteopenia and cachexia are considered emerging important syndromes for dogs and cats [10-12]. Several authors suggested biological markers for sarcopenia in dogs; among others, creatine phosphokinase and aspartate aminotransferase were suggested by Tvarijonaviciute, [13]. Sarcopenia is the decline of muscle function, which is frequently observed in elderly both in humans and animals. Sarcopenia is the results of different and interacting biological mechanisms.

The International European Working Group on Sarcopenia defined sarcopenia as a progressive and widespread skeletal muscle disorder, which is associated with a higher probability of morbidity such as falls, fractures, physical disability and mortality [14]. In humans after 30 years of age, strength and skeletal muscle mass gradually decrease every year by approximately 1 to $2 \%$ [15].

Some authors $[16,17]$ described very clearly how oxidative stress and chronic inflammation could contribute to sarcopenia; in fact, some pathways can be activated or inactivated during oxidative stress and inflammation. One of the critical steps of the suggested pathway is the increased production of damaged (oxidized) molecules, such as oxidized DNA, proteins and lipids. The oxidative processes that contribute to sarcopenia reduce satellite cell (myogenic stem cell) number and function. Satellite cell's function is affected by oxidative stress, which is elevated in aged muscles. Nutritional intervention could be a relatively new strategy to improve the satellite cell environment and systemic factors, with the objective to optimize satellite cell function in aging muscles [18]. there Several molecules have been proposed as biomarkers for oxidative stress. For their easy quantification, some of them seem particularly interesting to evaluate this state in dogs: alkaline phosphatase for the speed of bone remodeling [19] and 8-hydroxy-deoxy-guanosine $(8(\mathrm{OH}) \mathrm{dG})$ as a marker of DNA damage $[20,21]$.
On these bases, the aim of this study was the evaluation of the possible protective effects of a nutritional intervention on oxidative stress and, consequently, on the incidence of sarcopenia. Among the possible dietetic solutions, a product containing resveratrol and $\boldsymbol{\alpha}$-tocopherol acetate was selected for the supplementation, thanks to its known strong activity as an antioxidant, [22], [30].

\section{Materials and methods}

\subsection{Animals}

Dogs included in this study were 22; they were medium-sized adults $(5.4 \pm 1.4$ years old), including 10 males and 12 females, castrated and wormed; their weight was $19.4 \pm 4.4 \mathrm{~kg}$ (average \pm standard deviation). Animals were free from diseases as established by clinical examination and biochemical assays. The dogs of this study were identified with photographic and descriptive cards also supported by microchips compatible with all ISO standards DATAMARS brand. The microchips were placed subcutaneously in the area of the cross (at the beginning of the chest or the end of the neck). Dogs were maintained in the same hygienic conditions and diet with or without addition of the food supplement object of the study. No other medication or food supplement was allowed. At the day 0 of the study (D0) a baseline clinical examination was performed; a sample of venous blood was withdrawn for biochemical assays. During the following 45 days, dogs received twice a day the supplement, whose composition is reported in Table 1. Dogs received a dose/meal of $5 \mathrm{~g}$ of JUVENIA ${ }^{\circledR}$ DOGS (Microsules Argentina SA Laboratory; US patent required: 62659885), containing 30 $\mathrm{mg}$ of resveratrol and $20 \mathrm{UI}$ of $\boldsymbol{\alpha}$-tocopherol acetate; the latter corresponds to $4 \%$ of the recommended daily dose of Vitamin $E$ for middle-sized dogs At the end of the treatment period (day 45, D45), new clinical examinations and analysis of venous blood were performed. From the day 45 to day 90 (D45-D90), all dogs maintained the previous general conditions, but the diet did not include the supplementation. At D90, new examinations and collection of venous blood samples were performed.

\subsection{Methods}

\subsubsection{Measurement of DNA/RNA oxidative damage}

Both DNA and RNA are damaged by oxidation during aging and in a variety of disease states. The 
Table 1

Composition of the dietary supplement JUVENIA ${ }^{\circledR}$ DOGS

\begin{tabular}{lll}
\hline Compound & $\begin{array}{c}\text { Composition } \\
\%\end{array}$ & $\begin{array}{c}\text { Quantity (mg) } \\
\text { packet 5g }\end{array}$ \\
\hline Hydrolyzed bird liver & 54,15 & 2706 \\
Resveratrol & 0.60 & 30 \\
Vitamin E as acetate DL & 0.80 & 40 Equivalent \\
$\quad \alpha$ tocopherol 50\% & & to 20 UI \\
Chicken fat & 38,36 & 1920 \\
Water & 5,4 & 270 \\
Tween 80 & 0,56 & 28 \\
Nipagin sodium & 0,108 & 5,4 \\
Nipasol sodium & 0,028 & 1,4 \\
\hline
\end{tabular}

Composition of dietary supplement based on JUVENIA ${ }^{\circledR}$ DOGS. In the first column is shown, the composition in active principles; in the second column it is shown, in percentages; The third column shows, the amounts per dose/meal administered to each dog.

repair processes that are initiated to correct this damage release multiple oxidized guanine species into the blood and urine. An immunoassay (DNA/RNA Oxidative Damage kit) from Cayman Chemical (Cayman Chemical item No.589320). was used to quantify biomarkers of DNA/RNA oxidative damage: all three oxidized guanine species; 8-hydroxy-2'deoxyguanosine from DNA, 8-hydroxyguanosine from RNA, and 8-hydroxyguanine from either DNA or RNA. The serum of the dogs were maintained at $-80^{\circ} \mathrm{C}$ and analyzed according to the kit instructions.

Creatine phosphokinase (CPK) was quantified by Creatine Kinase assay on the ARCHITECT $c$ Systems $^{\mathrm{TM}}$ and the AEROSET System (Abbot Clinical Chemistry).

Total Alkaline Phosphatase (ALP) and bone subfraction (BAP) was quantified by spectrophotometry (Kit ALP - AMP Biosystems).

Statistics: The statistical analysis was performed using the Student $t$-test for paired samples (Prim 7.0, GraphPad, San Diego, CA, USA). The Student $t$-test was selected to evaluate a single biomarker at a time, in 3 series of paired data (D0 vs D45, D45 vs D90 and D0 vs D90) of each dog before and after the nutritional intervention.

\section{Results and discussion}

In this trial, animals were observed for two consecutive periods of 45 days, where the basic diet was added or not with a food supplement containing antioxidants $(30 \mathrm{mg}$ resveratrol and $20 \mathrm{UI}$ $\boldsymbol{\alpha}$-tocopherol acetate). The inclusion of the same dogs in the control and treated group minimizes the possibility of bias, such as intraspecies or interindividual variability.

As reported above, creatinine phosphokinase is considered a good biomarker for sarcopenia in dogs.

Regarding the effects observed on alkaline phosphatase, our hypothesis is that the nutritional intervention can modify the speed of bone remodeling, modulating the balance between the bone formation and resorption. In the absence of liver disease or pregnancy, alkaline phosphatase is an indirect biomarker of bone formation; since blood sampling was performed always at the same hour, the bone remodelling was evaluated without interference by the circadian rhythm. Different investigations showed that after starting a treatment with drugs to decrease bone resorption, there is a significant decrease in both the resorption biomarkers (between 4 to 6 weeks), and in bone formation biomarkers (between 2 and 3 months). In a large number of cases a valley value is reached at 2 or 3 months from the beginning of treatment and remains constant while treatment continues. Therefore, the modifications will depend on the therapeutic agent used and the biomarker studied. [23-24-25]. In young subjects, a high alkaline phosphatase value indicates that there is bone remodeling with predominance of formation; in adults with oxidative or postmenopausal stress, alkaline phosphatase is still high, but remodeling is associated with a net predominance of resorption [19].

From the results of statistical elaboration, it has been shown that food supplementation with antioxidants (resveratrol and $\boldsymbol{\alpha}$-tocopherol acetate) can modulate positively these biomarkers. As shown in Table 2, after 45 days of supplementation, there was a statistically significant reduction of serum concentrations of creatin (phospho) kinase (Fig. 1) and total alkaline phosphatase (Fig. 2).

The changes in alkaline phosphatase values were different in males and females: the basal values of alkaline phosphatase were higher in females, where the nutritional intervention influenced significantly the rate of bone remodeling at D45. Males showed a decrease in alkaline phosphatase concentration at the same time, but statistical significance was not reached. After the withdraw of the supplementation, a significant increase in the rate of bone remodeling was observed at Day 90 in both subgroups (Fig. 4 and Fig. 5). All the dogs were castrated and free from liver disease, therefore the decrease observed in Total Alkaline Phosphatase values at D45, should be due to the decrease in the speed of bone remodeling as 
Table 2

Results. The values expressed are the mean \pm standard deviation

\begin{tabular}{|c|c|c|c|c|c|c|}
\hline Biomarker & Day 0 & Day 45 & D 90 & $\begin{array}{c}P \\
\text { Day } 45 \text { vs } \\
\text { Day } 0\end{array}$ & $\begin{array}{c}P \\
\text { Day } 90 \text { vs } \\
\text { Day } 45\end{array}$ & $\begin{array}{c}P \\
\text { Day } 90 \mathrm{vs} \\
\text { Day } 0\end{array}$ \\
\hline Creatine (phospho) kinase (CPK) UI/L & $252 \pm 107.1$ & $176.5 \pm 69.4$ & $218.4 \pm 67.9$ & $<0.01$ & $<0.05$ & NS \\
\hline $\begin{array}{l}\text { Total Alkaline phosphatase (AP). Sub } \\
\text { fraction bone (BAP) was average } 82 \% \\
\text { of the AP. UI/L }\end{array}$ & $99.3 \pm 43.0$ & $76.3 \pm 27.3$ & $140.7 \pm 93.7$ & $<0.05$ & $<0.01$ & NS \\
\hline Total Alkaline phosphatase (AP) males & $87,9 \pm 40.9$ & $85,8 \pm 36.5$ & $132 \pm 52.5$ & NS & $<0,05$ & $<0,05$ \\
\hline Total Alkaline phosphatase (AP) females & $103,5 \pm 46,67$ & $74,41 \pm 24,77$ & $144,90 \pm 116.87$ & $<0,05$ & $<0,05$ & NS \\
\hline
\end{tabular}

The concentration of the serum biomarkers of sarcopenia (Creatin phosphokinase), bone remodeling (alkaline phosphatase), and DNA damage $(8(\mathrm{OH}) \mathrm{dG})$ evaluated at the end of each period is shown. Basal on day 0 , end of day 45 (with resveratrol and $\boldsymbol{\alpha}$-tocopherol acetate) and end of day 90 (without resveratrol and $\boldsymbol{\alpha}$-tocopherol acetate). It was significant in the comparison of the results of day 45 versus day 0 . Same as the results of day 90 versus day 45 . The evidence is that the supplement containing resveratrol and $\boldsymbol{\alpha}$-tocopherol acetate produced a positive modulation of sarcopenia, remodeling bone and oxidative DNA damage.

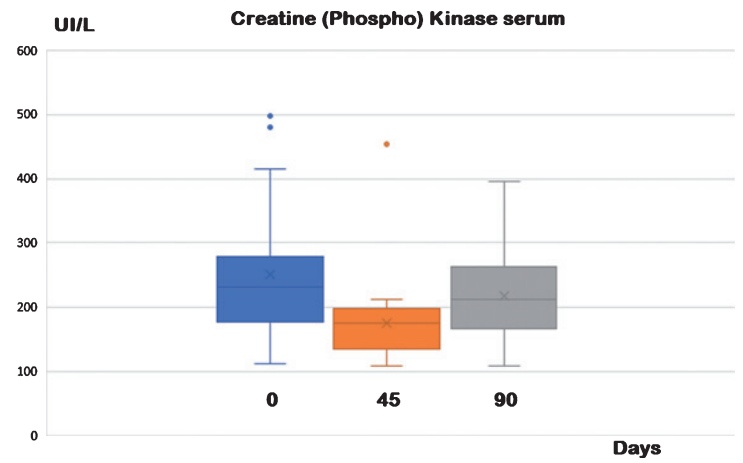

Fig. 1. Serum Creatine phosphokinase at different times and treatments. Animals were observed for two consecutive periods of 45 days, where the basic diet was added or not with a food supplement containing antioxidants (30 $\mathrm{mg}$ resveratrol and $20 \mathrm{UI} \boldsymbol{\alpha}$-tocopherol acetate). Creatin phosphokinase is considered a good biomarker for sarcopenia in dogs. After 45 days of supplementation, there was a statistically significant reduction of serum concentrations of creatin phosphokinase, (see Table 2). These values returned to baseline after a further 45 days of basal diet without supplementation (control).

a result of the antioxidant activity of the nutritional supplementation with Resveratrol and $\alpha$-Tocopherol. This hypothesis was confirmed by fact that values measured at D90, after 45 days of diet without supplementation (control), returned at at baseline. Also, in this case the increase was statistically significant, while no difference was observed between baseline and data at day 90 (Table 2). Although not statistically significant, a reduction of $13 \%$ of 8 -Hydroxy-deoxyguanosine $[8(\mathrm{OH}) \mathrm{dG}]$ was also observed between day 0 and $45(3266.7 \pm 1966$ versus $2856.1 \pm 1380.6$ $\mathrm{pg} / \mathrm{mL}, p=0.4273$ ); the high variability (standard deviation) of data was probably responsible for this result (Fig. 3). Moreover, oxidative processes on DNA probably need longer periods of treatment.

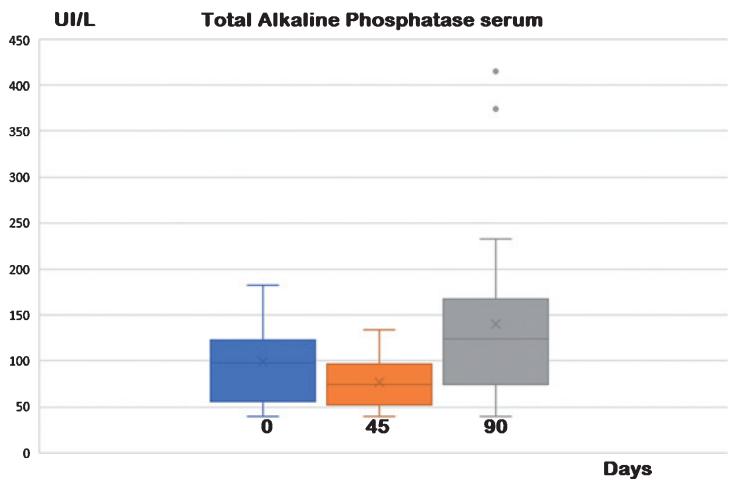

Fig. 2. Serum Alkaline Phosphatase at different times and treatments. Animals were observed for two consecutive periods of 45 days, where the basic diet was added or not with a food supplement containing antioxidants (30 mg resveratrol and $20 \mathrm{UI} \boldsymbol{\alpha}$-tocopherol acetate). From the results of statistical elaboration, it has been shown that food supplementation with antioxidants (resveratrol and $\boldsymbol{\alpha}$-tocopherol acetate) can modulate positively the alkaline phosphatase. After 45 days of supplementation, there was a statistically significant reduction of serum concentrations of total alkaline phosphatase. These values returned to baseline after a further 45 days of basal diet without supplementation (control). Also, in this case the increase was statistically significant, while no difference was observed between baseline and data at day 90, (see Table 2).

Urquiaga I. et al. [26] showed that moderate wine consumption (containing resveratrol) to both an occidental or a Mediterranean diet increases plasma antioxidant capacity, decreases oxidative DNA damage, and normalizes endothelial function. The results were more significant in the group consuming occidental diet. The improvement of feeding and veterinary care has allowed a considerable extension of the life span of pet animals, and of dogs and cats. Therefore, dogs show more frequently a decreasing ability in reacting to stress, a more fre- 


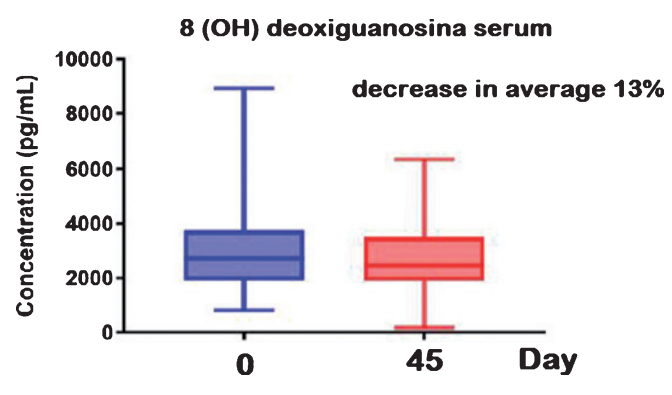

Fig. 3. Serum $8(\mathrm{OH}) \mathrm{dG}$ at different times and treatments. Animals were observed for two consecutive periods of 45 days, where the basic diet was added or not with a food supplement containing antioxidants (30 $\mathrm{mg}$ resveratrol and $20 \mathrm{UI} \boldsymbol{\alpha}$-tocopherol acetate). Although not statistically significant, a $13 \%$ reduction in the mean of 8-hydroxy-deoxy-guanosine $(8(\mathrm{OH}) \mathrm{dG})$ was also observed between days 0 and 45 (see Table 2). The high variability (standard deviation) of data was probably responsible for this result. Moreover, positive changes in DNA oxidation probably need longer periods of treatment and are also modulated by the basal diet used.

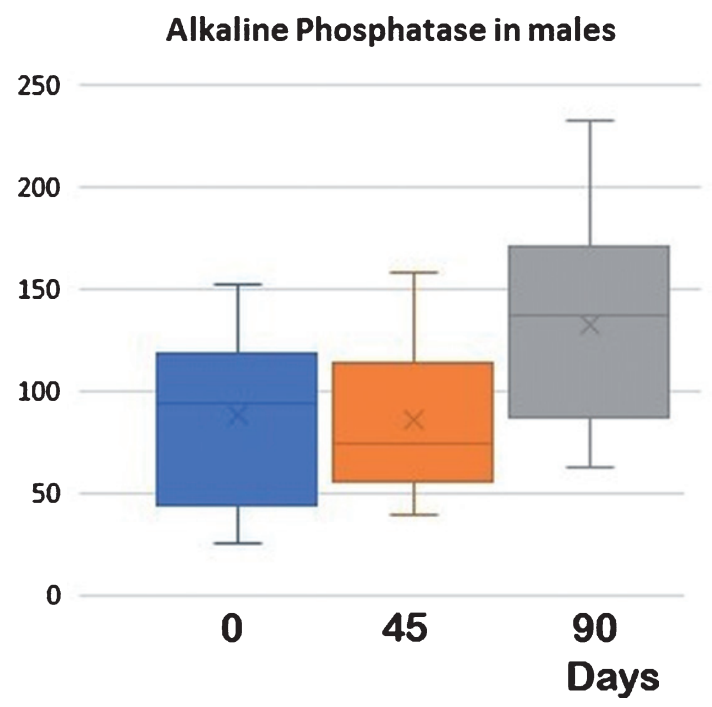

Fig. 4. Alkaline Phosphatase in males at different times and treatments. D0 vs D45 NS: D45 vs D90<0.05: D0 vs D $90<0.05$. Serum alkaline phosphatase in males. After 45 days of supplementation, serum concentrations of total alkaline phosphatase decreased but did not reach statistical significance. These values returned to the beginning after 45 additional days of basal diet without supplementation (control). In this case, the increase in values from D45 to D90 was statistically significant, whereas no differences were observed between the baseline and the data on day 90 , (see Table 2).

quent homeostatic imbalance and an increased risk of diseases. There is a body of scientific evidences that nutrition influences the epigenome. This dynamic relationship between nutrition and genes throughout the life of an organism has been recognized as

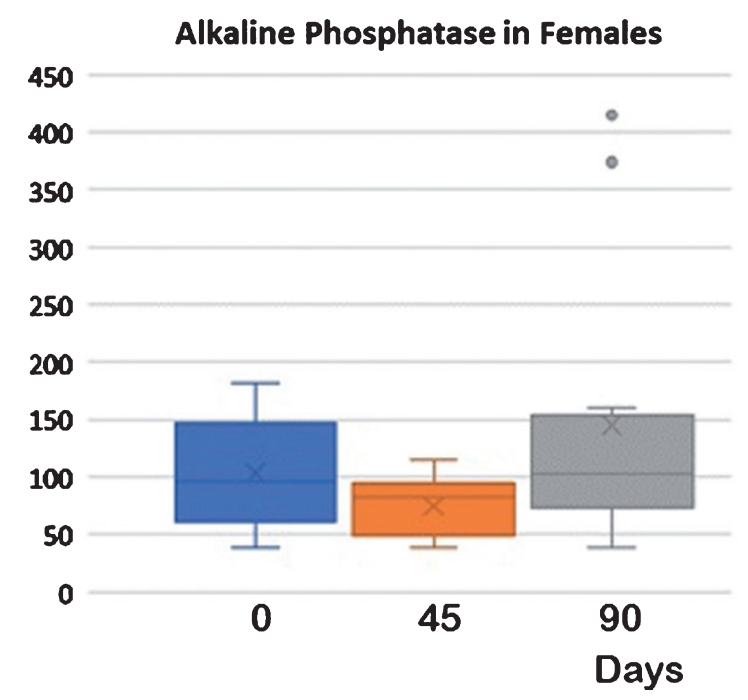

Fig. 5. Alkaline Phosphatase in females at different times and treatments. D0 vs D45<0,05: D45 vs D90<0,05: D0 vs D90 NS Serum alkaline phosphatase in females. After 45 days of supplementation, there was a statistically significant reduction of serum concentrations of alkaline phosphatase These values returned to baseline after a further 45 days of basal diet without supplementation (control). Also, in this case the increase was statistically significant, while no difference was observed between baseline and data at day 90, (see Table 2).

a subfield called Nutritional Epigenomic or Nutrigenomics. It provides promising information to improve health and prevent diseases [27-29].

Numerous evidences show the decrease of oxidative stress and sarcopenia by resveratrol supplementation, thanks to an increasing mitochondrial biogenesis, an improved control of the mitochondrial fusion/fission quality, an increase of the synthesis of antioxidant enzymes via SIRT1, and the effects similar to that of caloric restriction [30-33]. Some studies have shown that the antioxidant effect of $\alpha$ tocopherol acetate is directly correlated to a decrease in intracellular oxidative stress and improvement of strength and muscle mass [34]. This research on dogs was conducted in a biology that ages approximately 7 times faster than people [35] and supports the hypothesis that sarcopenia and the increased speed of bone remodeling, which frequently affect older dogs, can be at least partially prevented by the supplementation with antioxidants. The present study, we believe opens interesting questions for future research in the field of human aging and animals that would require longer intervention times with Resveratrol alone or in combination with other polyphenols. In fact, the supplementation with Resveratrol and $\boldsymbol{\alpha}$-tocopherol acetate caused a reduction of serum concentration 
in $\mathrm{CPK}$ and AP, which can be associated with the pathologies considered above in adult dogs. The correlation between supplementation and effect was confirmed by the second treatment period, in which the values of the two biomarkers returned to the baseline.

In conclusion, the present investigation has shown that the supplementation of the diet with a formula containing resveratrol and $\boldsymbol{\alpha}$-tocopherol acetate can modulate serum biomarkers, such as CPK and AP, which are associated with sarcopenia and increased speed of bone remodelling. Since these conditions accelerate aging and are increasing in the dog population due to the longer life span, the use of antioxidant supplementation could be useful in improving the quality of life of pets.

\section{Acknowledgments}

We would like to thank Microsules Argentina SA for the contribution of the JUVENIA ${ }^{\circledR}$ DOGS dietary supplement, that allowed us to carry out this study.

\section{Funding}

The authors report no funding.

\section{Conflict of interests}

The Authors declares that there is no conflict of interest.

\section{References}

[1] Sandra H. van Oostrom, Ronald Gijsen, Irina Stirbu, Joke C Korevaar, Francois G. Schellevis, H. Susan J Picavet1, Nancy Hoeymans. Time trends in prevalence of chronic diseases and multimorbidity not only due to aging: data from general practices and health surveys. PLoS One. 2016;11:e0160264.

[2] Moylan, J.S. and Reid M.B. Oxidative stress, chronic disease, and muscle wasting. Muscle Nerve 2007;35:411-29.

[3] Lien Ai Pham-Huy, Hua He, Chuong Pham-Huy. Free radicals, antioxidants in disease and health. Int J Biomed Sci. 2008;4:89-96.

[4] Sies H, Berndt C, Jones DP. Oxidative Stress. Annu Rev Biochem. 2017;86:715-48.

[5] Lobo V.A., Patil, A., Phatak, N., Chandra 2010. Free radicals, antioxidants and functional foods: Impact on human health. Pharmacogn Rev. 2010;4:118-26.

[6] Birben E., Umit Murat Sahiner, Cansin Sackesen, Serpil Erzurum, Omer Kalayci. Oxidative stress and antioxidant defence. World Allergy Organ J. 2012;5:9-19.
[7] Clifford J and Rosen MD (Editors). Prime on the metabolic Bone Diseases and Disorders of Mineral Metabolism. Washington D.C.: The American Society for Bone and Mineral Research; 2013.

[8] Almeida M., Li Han, Marta Martin-Millan, Lilian I. Plotkin, Scott A. Stewart, Paula K. Roberson, Stavroula Kousteni, Charles A. O'Brien, Teresita Bellido, A. Michael Parfitt, Robert S. Weinstein, Robert L. Jilka, and Stavros C. Manolagas. Skeletal involution by age-associated oxidative stress and its acceleration by loss of sex steroids. J Biol Chem. 2007;282:27285-97.

[9] Mederle OA., Melania Balas, Sorin Dumitru Ioanoviciu, Camelia-Vidita Gurba, Anca Tudor, Claudia Borza. Correlations between bone turnover markers, serum magnesium and bone mass density in postmenopausal osteoporosis. Clinical Interventions in Aging. 2018;13:1383-9.

[10] Calvani R, Federico Marini, Matteo Cesari, Matteo Tosato, Stefan D. Anker, Stephan von Haehling, Ram R. Miller, Roberto Bernabei, Francesco Landi, Emanuele Marzetti \& the SPRINTT consorti. Biomarkers for physical frailty and sarcopenia: state of the science and future developments. Journal of Cachexia Sarcopenia and Muscle. 2015;6:278-86.

[11] Freeman LM. Cachexia and sarcopenia: emerging syndromes of importance in dogs and cats. J Vet Intern Med. 2012;26: 3-17.

[12] Vlasakova, K., Pamela Lane, Laura Michna, Nagaraja Muniappa, Frank D. Sistare, and Warren E. Glaab. Response of novel skeletal muscle biomarkers in dogs to drug induced skeletal muscle injury or sustained endurance exercise. Toxicological Sciences. 2017;156:422-7.

[13] Tvarijonaviciute A., Tomas Barranco, Monica Rubio, Jose Maria Carrillo, Silvia Martinez-Subiela, Fernando Tecles, Juana Dolores Carrillo, José J. Cerón. Measurement of creatine kinase and aspartate amino transferase in saliva of dogs: a pilot study. BMC Veterinary Research. 2017;13:168.

[14] Cruz-Jentoft AJ., Gülistan Bahat, Jürgen Bauer, Yves Boirie, Olivier Bruyére, Tommy Cederholm, Cyrus Cooper, Francesco Landi, Yves Rolland, Avan Aihie Sayer, Stéphane M. Schneider, Cornel C. Sieber, Eva Topinkova, Maurits Vandewoude, Marjolein Visser, Mauro Zamboni, Writing Group For The European Working Group on Sarcopenia In Older People (EWGSOP2), And The Extendend Group For EWGSOP. Sarcopenia: revised European consensus on definition and diagnosis. Age and Ageing. 2019;48:16-31.

[15] Sehl ME, and F. Eugene Yates. Kinetics of human aging: I. Rates of senescence between ages 30 and 70 years in healthy people. The Journals of Gerontology A: Biological Sciences and Medical Sciences. 2001;56:B198-B208.

[16] Meng S.J., and Long-Jiang Yu. Oxidative stress, molecular inflammation and sarcopenia. Int J Mol Sci. 2010;11: 1509-26.

[17] Brioche T, Lemoine-Morel S. Oxidative stress, sarcopenia, antioxidant strategies and exercise: Molecular aspects. Current Pharmaceutical Design. 2016;22:2664-78.

[18] Alway Stephen E, Matthew J. Myers, Junaith S. Mohamed. Regulation of satellite cell function in sarcopenia. Frontiers in Aging Neuroscience. 2014; Volume 6:Article 246.

[19] Romagnoli Elisabetta, Giovanni Minisola, Vincenzo Carnevale, Alfredo Scillitani, Vincenzo Frusciante, Giuseppe Aliberti, Salvatore Minisola. Assessment of Serum Total and Bone Alkaline Phosphatase Measurement in Clinical Practice Clin Chem Lab Med. 1998;36:163-8. 
[20] Wu Lily L., Chiuan-Chian Chiou, Pi-Yueh Chang, James T. Wu. Urinary 8-OHdG: a marker of oxidative stress to DNA and a risk factor for cancer, atherosclerosis and diabetics. Clin Chim Acta. 2004;339:1-9.

[21] Wu Danni, Baodong Liu, Junfa Yin, Tian Xu, Shuli Zhao, Qun $\mathrm{Xu}, \mathrm{Xi}$ Chen, Hailin Wang. Detection of 8-Hydroxy deoxy-guanosine $[8(\mathrm{OH}) \mathrm{dG}]$ as a biomarker of oxidative damage in peripheral leukocyte DNA by UHPLC-MS/MS. J Chromatography B. 2017;1064:1-6.

[22] Gülçin İlhami. Antioxidant properties of resveratrol: A structure-activity insight. Innovative Food Science and Engirering Technologies. 2010;11:210-8.

[23] Romero Barco Carmen M., Sara Manrique Arija, Manuel Rodriguez Perez. Marcadores bioquímicos en osteoporosis. Utilidad en la práctica clínica. Reumatol Clin. 2012; 8:149-52.

[24] Delmas PD. Committee of Scientific Advisors of the International Osteoporosis Foundation. The use of biochemical markers of bone turnover in osteoporosis. Osteoporos Int. 2000;11(Suppl 6):2-17.

[25] Singer FR, Eyre DR. Using biochemical markers of bone turnover in clinical practice. Cleve Clin J Med. 2008; 75:739-50.

[26] Urquiaga Inés, Pablo Strobel, Druso Perez, Carlos Martinez, Ada Cuevas, Oscar Castillo, Guillermo Marshall, Jaime Rozowski, Federico Leighton. Mediterranean diet and red wine protect against oxidative damage in youngs volunteers. Atherosclerosis. 2010;211:694-9.

[27] Fang Mingzhu, Dapeng Chen, and Chung S. Yang. Dietary polyphenols may affect DNA methylation. J Nutr. 2007;137:223S-228S.

[28] Reik Wolf. Stability and flexibility of epigenetic gene regulation in mammalian development. Nature. 2007;447: 425-432.

[29] Zhang Naifeng. Epigenetic modulation of DNA methylation by nutrition and its mechanisms in animals. Animal Nutrition. 2015;1(3):144-51.
[30] Price Nathan L, Ana P. Gomes, Alvin J.Y. Ling, Filipe V. Duarte, Alejandro Martin-Montalvo, Brian J. North, Beamon Agarwal, Lan Ye, Giorgio Ramadori, Joao S. Teodoro, Basil P. Hubbard, Ana T. Varela, James G. Davis, Behzad Varamini, Angela Hafner, Ruin Moaddel, Anabela P. Rolo, Roberto Coppari, Carlos M. Palmeira, Rafael de Cabo, Joseph A. Baur, and David A. Sinclair. SIRT1 is Required for AMPK Activation and the Beneficial Effects of Resveratrol on Mitochondrial Function. Cell Metabolism. 2012;15:675-90.

[31] Lagouge Marie, Carmen Argmann, Zachary Gerhart-Hines, Hamid Meziane, Carles Lerin, Frederic Daussin, Nadia Messadeq, Jill Milne, Philip Lambert, Peter Elliott, Bernard Geny, Markku Laakso, Pere Puigserver, and Johan Auwerx. Resveratrol Improves Mitochondrial Function and Protects against Metabolic Disease by Activating SIRT1 and PGC-1 $\alpha$. Cell. 2006;127:1109-22.

[32] Hu Yi, Jing Liu, Junfeng Wang, Qingsong Liu. The controversial links among calorie restriction, SIRT1, and resveratrol. Free Radical Biology \& Medicine. 2011;51:250-6.

[33] Kuršvietienè Lolita, Inga Stanevičiene, Aušra Mongirdienė, Jurga Bernatonienè. Multiplicity of effects and health benefits of resveratrol. Medicina. 2016;52:148-55.

[34] Khor Shy Cian, Norwahidah Abdul Karim, Wan Zurinah Wan Ngah, Yasmin Anum Mohd Yusof, Suzana Makpol. Vitamin E in Sarcopenia: Current Evidences on Its Role in Prevention and Treatment. Oxidative Medicine and Cellular Longevity. Volume 2014, Article ID 914853, 16 pages http://dx.doi.org/10.1155/2014/914853.

[35] Matt Kaeberlein, Kate E. Creevy, Daniel E.L. Promislow. The Dog Aging Project: Translational Geroscience in Companion Animals. Mamm Genome. 2016;27(7-8):279-88. 